\title{
Fourier series of higher-order ordered Bell functions
}

\author{
Taekyun Kim, ${ }^{\mathrm{a}, \mathrm{b}}$, Dae San Kimc, Gwan-Woo Jang ${ }^{\mathrm{b}}$, Jongkyum Kwon ${ }^{\mathrm{d}, *}$ \\ ${ }^{a}$ Department of Mathematics, College of Science, Tianjin Polytechnic University, Tianjin 300160, China. \\ ${ }^{b}$ Department of Mathematics, Kwangwoon University, Seoul, 139-701, Republic of Korea. \\ ${ }^{c}$ Department of Mathematics, Sogang University, Seoul, 121-742, Republic of Korea. \\ ${ }^{d}$ Department of Mathematics Education and RINS, Gyeongsang National University, Jinju, Gyeongsangnamdo, 52828, Republic of \\ Korea.
}

Communicated by S.-H. Rim

\begin{abstract}
In this paper, we consider higher-order ordered Bell functions and derive their Fourier series expansions. Moreover, we express those functions in terms of Bernoulli functions. (C)2017 All rights reserved.
\end{abstract}

Keywords: Fourier series, higher-order ordered Bell functions, higher-order ordered Bell polynomials. 2010 MSC: 11B83, 42A16.

\section{Introduction}

For $r \in \mathbb{Z}_{>0}$, the ordered Bell polynomials $b_{m}^{(r)}(x)$ of order $r$ are defined by the generating function

$$
\left(\frac{1}{2-e^{t}}\right)^{r} e^{x t}=\sum_{m=0}^{\infty} b_{m}^{(r)}(x) \frac{t^{m}}{m !} .
$$

When $x=0, b_{m}^{(r)}=b_{m}^{(r)}(0)$ are called the ordered Bell numbers of order $r$. In particular, $b_{m}(x)=b_{m}^{(1)}(x)$ and $b_{m}=b_{m}^{(1)}$ are respectively called the ordered Bell polynomials and the ordered Bell numbers.

The first appearance of the ordered Bell numbers $b_{m}$ goes back to as early as 1859 , when Cayley used them to count certain plane trees with $m+1$ totally ordered leaves. Since then, they have been studied in many counting problems in number theory and enumerative combinatorics (see $[2,3,5,8,12,14,15]$ ). The ordered Bell numbers $b_{m}$ are all positive integers, as we can see from

$$
b_{m}=\sum_{n=0}^{m} n ! S_{2}(m, n)=\sum_{n=0}^{\infty} \frac{n^{m}}{2^{n+1}}, \quad(m \geqslant 0) .
$$

\footnotetext{
${ }^{*}$ Corresponding author

Email addresses: tkkim@kw.ac.kr (Taekyun Kim), dskim@sogang.ac.kr (Dae San Kim), jgw5687@naver.com (Gwan-Woo Jang), mathkjk26@gnu.ac.kr (Jongkyum Kwon)

doi:10.22436/jnsa.010.07.39
} 
On the other hand, the ordered Bell polynomial $b_{m}(x)$ has degree $m$ and is a monic polynomial with integral coefficients, as we can see, for example, from

$$
b_{0}(x)=1, b_{m}(x)=x^{m}+\sum_{l=0}^{m-1}\left(\begin{array}{c}
m \\
l
\end{array}\right) b_{l}(x), \quad(m \geqslant 1) .
$$

From (1.1), we can derive

$$
\frac{d}{d x} b_{m}^{(r)}(x)=m b_{m-1}^{(r)}(x), \quad(m \geqslant 1), \quad b_{m}^{(r)}(x+1)-b_{m}^{(r)}(x)=b_{m}^{(r)}(x)-b_{m}^{(r-1)}(x), \quad(m \geqslant 0) .
$$

In turn, from these we obtain

$$
\begin{aligned}
& b_{m}^{(r)}(1)-b_{m}^{(r)}=b_{m}^{(r)}-b_{m}^{(r-1)}, \quad(m \geqslant 0), \\
& \int_{0}^{1} b_{m}^{(r)}(x) d x=\frac{1}{m+1}\left(b_{m+1}^{(r)}(1)-b_{m+1}^{(r)}\right)=\frac{1}{m+1}\left(b_{m+1}^{(r)}-b_{m+1}^{(r-1)}\right) .
\end{aligned}
$$

As is well-known, the Bernoulli polynomials $B_{m}(x)$ are given by the generating function

$$
\frac{t}{e^{t}-1} e^{x t}=\sum_{m=0}^{\infty} B_{m}(x) \frac{t^{m}}{m !} .
$$

For any real number $x$, let

$$
<x>=x-[x] \in[0,1)
$$

denote the fractional part of $x$.

The reader may refer to any book (for example, see $[1,13,16])$ for elementary facts about Fourier analysis. Also, we will need the following well-known facts about Bernoulli functions $B_{\mathfrak{n}}(<x>)$ :

(a) for $m \geqslant 2$,

$$
B_{m}(<x>)=-m ! \sum_{n=-\infty, n \neq 0}^{\infty} \frac{e^{2 \pi i n x}}{(2 \pi i n)^{m}}
$$

(b) for $m=1$,

$$
-\sum_{n=-\infty, n \neq 0}^{\infty} \frac{e^{2 \pi i n x}}{2 \pi i n}= \begin{cases}B_{1}(<x>), & \text { for } x \notin \mathbb{Z}, \\ 0, & \text { for } x \in \mathbb{Z} .\end{cases}
$$

Here we will consider the higher-order ordered Bell functions $b_{m}^{(r)}(<x>)$, and derive its Fourier series expansions. In addition, we will express those functions in terms of Bernoulli functions.

As to the higher-order ordered Bell functions $b_{m}^{(r)}(<x>)$, we note that the polynomial identity (1.3) follows immediately from Theorems 2.1 and 2.2, which can be derived in turn from the Fourier series expansion of $b_{m}^{(r)}(<x>)$,

$$
b_{m}^{(r)}(x)=\frac{1}{m+1} \sum_{j=0}^{m}\left(\begin{array}{c}
m+1 \\
j
\end{array}\right)\left(b_{m-j+1}^{(r)}-b_{m-j+1}^{(r-1)}\right) B_{j}(x) .
$$

Finally, the reader may refer to $[4,6,7,9-11]$ for some recent related works.

\section{Fourier series of higher-order ordered Bell functions}

From now on, we will assume that $m \geqslant 1$ and $r \geqslant 2$. The case of $r=1$ has been treated as a special case of the results in [4]. 
$b_{m}^{(r)}(<x>)$ is piecewise $C^{\infty}$. Moreover, in view of (1.2), $b_{m}^{(r)}(<x>)$ is continuous for those integers $(r, m)$ with $b_{m}^{(r)}=b_{m}^{(r-1)}$, and is discontinuous with jump discontinuities at integers for those $(r, m)$ with $\mathrm{b}_{\mathrm{m}}^{(\mathrm{r})} \neq \mathrm{b}_{\mathrm{m}}^{(\mathrm{r}-1)}$.

The Fourier series of $b_{m}^{(r)}(<x>)$ is

$$
\sum_{n=-\infty}^{\infty} A_{n}^{(r, m)} e^{2 \pi i n x}
$$

where

$$
A_{n}^{(r, m)}=\int_{0}^{1} b_{m}^{(r)}(<x>) e^{-2 \pi i n x} d x=\int_{0}^{1} b_{m}^{(r)}(x) e^{-2 \pi i n x} d x
$$

Now, we would like to determine the Fourier coefficients $A_{n}^{(r, m)}$.

Case $1: n \neq 0$.

For $r \geqslant 2$, and $m \geqslant 1$, we set

$$
\begin{aligned}
\Delta_{r, m} & =b_{m}^{(r)}(1)-b_{m}^{(r)}=b_{m}^{(r)}-b_{m}^{(r-1)} \\
A_{n}^{(r, m)} & =\int_{0}^{1} b_{m}^{(r)}(x) e^{-2 \pi i n x} d x \\
& =-\frac{1}{2 \pi i n}\left[b_{m}^{(r)}(x) e^{-2 \pi i n x}\right]_{0}^{1}+\frac{1}{2 \pi i n} \int_{0}^{1}\left(\frac{d}{d x} b_{m}^{(r)}(x)\right) e^{-2 \pi i n x} d x \\
& =-\frac{1}{2 \pi i n}\left(b_{m}^{(r)}(1)-b_{m}^{(r)}\right)+\frac{m}{2 \pi i n} \int_{0}^{1} b_{m-1}^{(r)}(x) e^{-2 \pi i n x} d x \\
& =\frac{m}{2 \pi i n} A_{n}^{(r, m-1)}-\frac{1}{2 \pi i n} \Delta_{r, m} \\
& =\frac{m}{2 \pi i n}\left(\frac{m-1}{2 \pi i n} A_{n}^{(r, m-2)}-\frac{1}{2 \pi i n} \Delta_{r, m-1}\right)-\frac{1}{2 \pi i n} \Delta_{r, m} \\
& =\frac{(m)_{2}}{(2 \pi i n)^{2}} A_{n}^{(r, m-2)}-\sum_{j=1}^{2} \frac{(m)_{j-1}}{(2 \pi i n)^{j}} \Delta_{r, m-j+1} \\
& =\frac{(m)_{2}}{(2 \pi i n)^{2}}\left(\frac{m-2}{2 \pi i n} A_{n}^{(r, m-3)}-\frac{1}{2 \pi i n} \Delta_{r, m-2}\right)-\sum_{j=1}^{2} \frac{(m)_{j-1}}{(2 \pi i n)^{j}} \Delta_{r, m-j+1} \\
& =\frac{(m)_{3}}{(2 \pi i n)^{3}} A_{n}^{(r, m-3)}-\sum_{j=1}^{3} \frac{(m)_{j-1}}{(2 \pi i n)^{j}} \Delta_{r, m-j+1} \\
& \vdots \\
& =\frac{m !}{(2 \pi i n)^{m}} A_{n}^{(r, 0)}-\sum_{j=1}^{m} \frac{(m)_{j-1}}{(2 \pi i n)^{j}} \Delta_{r, m-j+1} \\
& =-\frac{1}{m+1} \sum_{j=1}^{m} \frac{(m+1)_{j}}{(2 \pi i n)^{j}} \Delta_{r, m-j+1} \cdot \\
&
\end{aligned}
$$

Case 2: $\mathfrak{n}=0$.

$$
A_{0}^{(r, m)}=\int_{0}^{1} b_{m}^{(r)}(x) d x=\frac{1}{m+1} \Delta_{r, m+1}
$$

Assume first that $\Delta_{r, m}=0$. Then $b_{m}^{(r)}(1)=b_{m}^{(r)}(0)$. As $b_{m}^{(r)}(<x>)$ is piecewise $C^{\infty}$ and continuous, the Fourier series of $b_{m}^{(r)}(<x>)$ converges uniformly to $b_{m}^{(r)}(<x>)$, and 


$$
\begin{aligned}
\mathrm{b}_{\mathrm{m}}^{(\mathrm{r})}( & <x>) \\
& =\frac{1}{\mathrm{~m}+1} \Delta_{r, m+1}+\sum_{n=-\infty, n \neq 0}^{\infty}\left(-\frac{1}{m+1} \sum_{j=1}^{m} \frac{(m+1)_{j}}{(2 \pi i n)^{j}} \Delta_{r, m-j+1}\right) e^{2 \pi i n x} \\
& =\frac{1}{m+1} \Delta_{r, m+1}+\frac{1}{m+1} \sum_{j=1}^{m}\left(\begin{array}{c}
m+1 \\
j
\end{array}\right) \Delta_{r, m-j+1} \times\left(-j ! \sum_{n=-\infty, n \neq 0}^{\infty} \frac{e^{2 \pi i n x}}{(2 \pi i n)^{j}}\right) \\
& =\frac{1}{m+1} \Delta_{r, m+1}+\frac{1}{m+1} \sum_{j=2}^{m}\left(\begin{array}{c}
m+1 \\
j
\end{array}\right) \Delta_{r, m-j+1} B_{j}(<x>)+\Delta_{r, m} \times \begin{cases}B_{1}(<x>), & \text { for } x \notin \mathbb{Z}, \\
0, & \text { for } x \in \mathbb{Z} .\end{cases}
\end{aligned}
$$

We are now ready to state our first theorem.

Theorem 2.1. For positive integers $r, l$ with $r \geqslant 2$, we let

$$
\Delta_{\mathrm{r}, \mathrm{l}}=\mathrm{b}_{\mathrm{l}}^{(\mathrm{r})}-\mathrm{b}_{\mathrm{l}}^{(\mathrm{r}-1)} \text {. }
$$

Assume that $\Delta_{r, m}=0$ for positive integers $r, m$ with $r \geqslant 2$. Then we have the following:

(a) $b_{m}^{(r)}(<x>)$ has the Fourier series expansion

$$
b_{m}^{(r)}(<x>)=\frac{1}{m+1} \Delta_{r, m+1}+\sum_{n=-\infty, n \neq 0}^{\infty}\left(-\frac{1}{m+1} \sum_{j=1}^{m} \frac{(m+1)_{j}}{(2 \pi i n)^{j}} \Delta_{r, m-j+1}\right) e^{2 \pi i n x}
$$

for all $x \in \mathbb{R}$, where the convergence is uniform;

(b)

$$
b_{m}^{(r)}(<x>)=\frac{1}{m+1} \Delta_{r, m+1}+\frac{1}{m+1} \sum_{j=2}^{m}\left(\begin{array}{c}
m+1 \\
j
\end{array}\right) \Delta_{r, m-j+1} B_{j}(<x>)
$$

for all $\mathrm{x} \in \mathbb{R}$, where $\left.\mathrm{B}_{\mathfrak{j}}(<\mathrm{x}\rangle\right)$ is the Bernoulli function.

Assume next that $\Delta_{r, m} \neq 0$ for positive integers $r, m$ with $r \geqslant 2$. Then $b_{m}^{(r)}(1) \neq b_{m}^{(r)}(0)$. Thus $b_{m}^{(r)}(<x>)$ is piecewise $C^{\infty}$, and discontinuous with jump discontinuities at integers. The Fourier series of $b_{m}^{(r)}(<x>)$ converges pointwise to $b_{m}^{(r)}(<x>)$ for $x \notin \mathbb{Z}$, and converges to

$$
\frac{1}{2}\left(b_{m}^{(r)}(0)+b_{m}^{(r)}(1)\right)=b_{m}^{(r)}+\frac{1}{2} \Delta_{r, m}
$$

for $x \in \mathbb{Z}$.

Now, we can state our second theorem.

Theorem 2.2. For positive integers $r, l$ with $r \geqslant 2$, we let

$$
\Delta_{\mathrm{r}, \mathrm{l}}=\mathrm{b}_{\mathrm{l}}^{(\mathrm{r})}-\mathrm{b}_{\mathrm{l}}^{(\mathrm{r}-1)}
$$

Assume that $\Delta_{r, m} \neq 0$ for positive integers $r, m$ with $r \geqslant 2$. Then we have the following:

(a)

$$
\frac{1}{m+1} \Delta_{r, m+1}+\sum_{n=-\infty, n \neq 0}^{\infty}\left(-\frac{1}{m+1} \sum_{j=1}^{m} \frac{(m+1)_{j}}{(2 \pi i n)^{j}} \Delta_{r, m-j+1}\right) e^{2 \pi i n x}= \begin{cases}b_{m}^{(r)}(<x>), & \text { for } x \notin \mathbb{Z}, \\ b_{m}^{(r)}+\frac{1}{2} \Delta_{r, m}, & \text { for } x \in \mathbb{Z} ;\end{cases}
$$


(b)

$$
\begin{aligned}
& \frac{1}{m+1} \sum_{j=0}^{m}\left(\begin{array}{c}
m+1 \\
j
\end{array}\right) \Delta_{r, m-j+1} B_{j}(<x>)=b_{m}^{(r)}(<x>), \text { for } x \notin \mathbb{Z}, \\
& \frac{1}{m+1} \sum_{\substack{j=0 \\
j \neq 1}}^{m}\left(\begin{array}{c}
m+1 \\
j
\end{array}\right) \Delta_{r, m-j+1} B_{j}(<x>)=b_{m}^{(r)}+\frac{1}{2} \Delta_{r, m}, \text { for } x \in \mathbb{Z} .
\end{aligned}
$$

\section{References}

[1] M. Abramowitz, I. A. Stegun, Handbook of mathematical functions with formulas, graphs, and mathematical tables, National Bureau of Standards Applied Mathematics Series, For sale by the Superintendent of Documents, U.S. Government Printing Office, Washington, D.C., (1964), 1

[2] A. Cayley, On the analytical forms called trees, Philos. Mag., 9 (1859), 374-378. 1

[3] L. Comtet, Advanced combinatorics, The art of finite and infinite expansions, Revised and enlarged edition, D. Reidel Publishing Co., Dordrecht, (1974). 1

[4] D. V. Dolgy, D. S. Kim, T. Kim, T. Mansour, Sums of finite products of ordered Bell functions, (preprint). 1, 2

[5] I. J. Good, The number of orderings of $n$ candidates when ties are permitted, Fibonacci Quart., 13 (1975), 11-18. 1

[6] G.-W. Jang, D. S. Kim, T. Kim, T. Mansour, Fourier series of functions related to Bernoulli polynomials, Adv. Stud. Contemp. Math., 27 (2017), 49-62. 1

[7] T. Kim, Euler numbers and polynomials associated with zeta functions, Abstr. Appl. Anal., 2008 (2008), 11 pages. 1

[8] T. Kim, D. S. Kim, On X-Bell polynomials associated with umbral calculus, Russ. J. Math. Phys., 24 (2017), 69-78. 1

[9] D. S. Kim, T. Kim, Fourier series of higher-order Euler functions and their applications, Bull. Korean Math. Soc., (to appear). 1

[10] T. Kim, D. S. Kim, Some formulas of ordered Bell numbers and polynomials arising from umbral calculus, (preprint).

[11] T. Kim, D. S. Kim, S.-H. Rim, D.-V. Dolgy, Fourier series of higher-order Bernoulli functions and their applications, J. Inequal. Appl., 2017 (2017), 7 pages. 1

[12] A. Knopfmacher, M. E. Mays, A survey of factorization counting functions, Int. J. Number Theory, 1 (2005), 563-581. 1

[13] J. E. Marsden, Elementary classical analysis, With the assistance of Michael Buchner, Amy Erickson, Adam Hausknecht, Dennis Heifetz, Janet Macrae and William Wilson, and with contributions by Paul Chernoff, István Fáry and Robert Gulliver, W. H. Freeman and Co., San Francisco, (1974). 1

[14] M. Mor, A. S. Fraenkel, Cayley permutations, Discrete Math., 48 (1984), 101-112. 1

[15] A. Sklar, On the factorization of squarefree integers, Proc. Amer. Math. Soc., 3 (1952), 701-705. 1

[16] D. G. Zill, W. R. Wright, M. R. Cullen, Advanced engineering mathematics, Fourth edition, Jones and Bartlett Publishers, Mississauga, Ontario, (2011). 1 